\title{
Acquired pragmatic disorders of right hemisphere damaged patients
}

Lívia Ivaskó ${ }^{1}$, Alinka Tóth ${ }^{2}$

${ }^{1}$ Developmental and Neuropragmatic Research Group, University of Szeged

${ }^{2}$ Department of Neurorehabilitation, University of Szeged

https://doi.org/10.36505/ExLing-2012/05/0017/000223

\begin{abstract}
In neuropragmatics attempts have been made to investigate how the brain/mind uses language both in healthy and neurologically impaired individuals (Paradis, 2009). This field, combining the approaches of linguistics and neurology, focuses on the communicative use of language, its neurological/neural basis and representation in the brain, mental strategies, communicative difficulties, intentional communication, inference from discourse and the role of context (contextual clues) in comprehension. Studies often deal with "pragmatic competence" (Perkins, 2010). By means of experimental pragmatics, our current research focuses on pragmatic competence and its vital component, the theory of mind (Baron-Cohen, 1985, 1995, 2000; Frith, 2007).
\end{abstract}

Key words: theory of mind, right hemisphere damage, pragmatic competence, intentionality, inference

\section{Hypotheses, materials and procedure}

It is assumed that the intentional use of language is affected by right hemisphere damage (RHD), and it is also proposed that there is dissociation between patients' pragmatic competence and linguistic performance (Chomsky, 1968; Paradis, 2009). This study supposes that there would not be noticeable differences in the performance of RHD patients and the members of the control group concerning their understanding of natural causality. The participants of the experiment were patients treated at the Department of Neurology and Neurosurgery at the University of Szeged. Experiments were conducted with 15 right frontal hemisphere damaged patients and 15 controls. The latter group consisted of healthy persons, whose sex, age and qualifications were the same as those of the test group. Firstly, we used the Hungarian version of the Western Aphasia Battery in order to assess the linguistic functions of the patients. Their abilities to understand intentionality were examined by means of a series of tasks compiled by us, a special picture test focusing on patients' ability to recognize and assume intentional connections between the pictures/sequences. Firstorder representational abilities, being able to describe what is happening in reality around us, are not enough to interpret certain implicatures or irony; in these cases one needs to rely on his second-order metarepresentational abilities to understand and consider the mental

ExLing 2012: Proceedings of 5th Tutorial and Research Workshop on Experimental Linguistics, 27-29 August 2012, Athens, Greece 
states of others (Happé, 2003). These pictures show stories including connections based on natural intentionality and others show human behaviour/ intentions. These pictures can be divided into four categories based on their content: representing natural causality, firs-order intentionality, irony. Each subtask included four pictures as elements of a story which the participants were asked to put into the correct order, and then they had to answer two testing questions. By means of these questions, it was possible to find out whether participants interpreted the stories properly, and on the other hand, it was vital to examine how the patients in the experiment attributed intentions to others. Sequencing and their answers to the questions (showing intention attribution) were scored separately within each task item. Data were analysed by means of SPSS software. Then a two-sample t-test was used to compare the differences in the performance of the two samples in the experiment. Furthermore, to be able to study the performance profile of the groups separately, we applied a paired t-test. Finally, we calculated percentages in those subtasks where the differences in the maximum scores did not enable us to carry out t-tests.

\section{Results and conclusion}

Comparing the performance of the two groups, it is obvious that the control group performed better in each task. Moreover, the members of the control group did not only do better, but their results are rather more homogeneous than those of the test group.

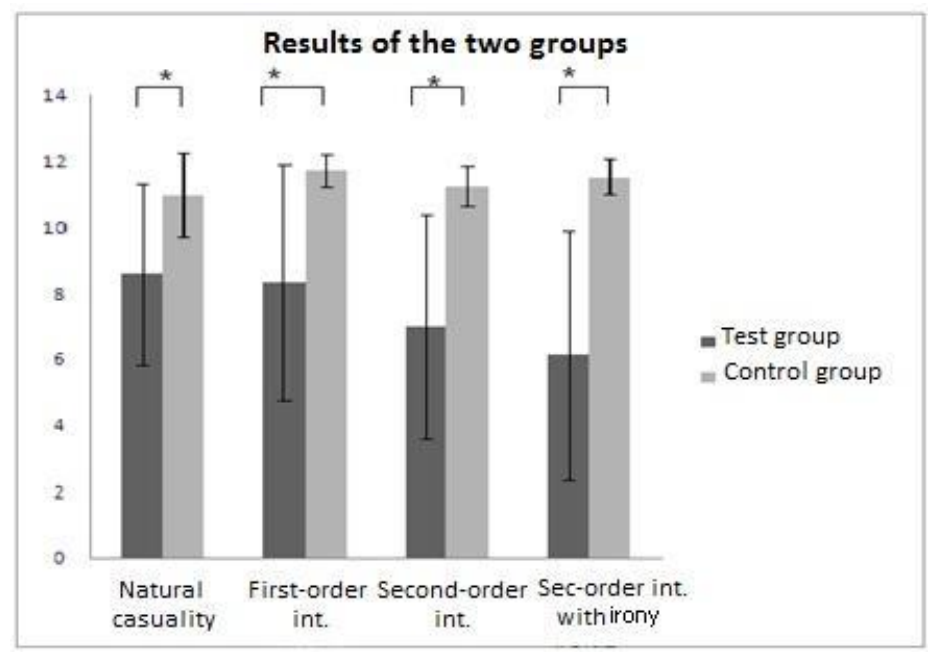

Figure 1: Total results of the two groups. 
The present experiment demonstrates that the executive functions are located in the frontal lobe (Frith, 2007). Considering the approach of Perkins (2010), these results also demonstrate the importance of further research in a wider context, as it seems that during communication, the abilities of understanding natural causality and the intentions of others complement one another, thus contributing to successful interpretation. Therefore, the main goal of the current experiment was to explore the relationship between inferential processes underlying the interpretation of natural causality and the so-called pragmatic inferential processes underlying the interpretation of intentional sequences. Analysing the scores of the intention attribution-tasks, there were significant differences between the points of RHD participants and the members of the control group. These findings confirm the emergentist approach to pragmatics, which defines pragmatic competence as dynamic relationship of several interconnected abilities and capacities (Perkins, 2010).

Subtasks focusing on the analysis of non-literal language included pictorial stories with irony and pictorial stories with idioms. While RHD participants had difficulties in understanding the idiomatic expressions embedded into the test, we could see the most significant differences when considering their comprehension of irony. To put it briefly, the test group achieved the lowest results in these tasks. These findings also demonstrate the fact that our ability to interpret irony appropriately greatly relies on our second-order metarepresentational abilities (Happé, 2003), which proves to be damaged in patients with brain lesions. We should draw attention to the fact that the performance of the participants in the test group did not decrease in parallel with the increasingly demanding nature of the tasks. In that case, the last task should have been the most difficult for the participants. Finally, the analysis of non-literal language indicates similar results concerning the interpretation of irony and idioms. The test group scored $51.08 \%$ and $56 \%$ in these tasks, while the control group scored $96.08 \%$ and $100 \%$. These percentages demonstrate the considerable differences between the performance of the two groups taking part in the experiment.

The current research also focuses on the question of localization. According to Frith-Wolpert (2003), injuries in the temporo-parietal and frontal cortex affect pragmatic performance and competence. Our research confirms the assumptions that several typical deficiencies are caused by right hemisphere damage, for example, discourse organization deficits, problems of cohesion, deriving the main point of a story, logical coherence, troubles with considering contextual clues. Individuals with RHD can make conscious logical deductions but cannot make implicit pragmatic inferences. They are unable to understand the so-called figurative meanings relying on others' cognitive competences, not just on the appropriate linguistic choices 
(Paradis, 2009). Our findings demonstrate that patients suffering from subcortical impairment do worse in these tasks than patients with cortical impairment. Although there is a need for a large-scale research to study this matter in detail, based on our results, we claim that white matter impairment, the damage of transmission pathways, can be seen as another cause of deficiencies in performance, such as the damage of neurons in the case of cortical impairment leading to functional disorder.

\section{Acknowledgements}

The authors of the article are grateful for the help of Prof. Dr. László Vécsei, the Dean of the Faculty of Medicine and the Director and Professor at the Department of Neurology and the help of Prof. Dr. Pál Barzó, the Director and Professor of the Department of Neurosurgery, and Dr. Katalin Jakab $\mathrm{PhD}$, Head of the Department of Neurorehabilitation (University of Szeged). Special thanks to Zsuzsanna Lengyel, the translator of the article.

\section{References}

Baron-Cohen, S., Leslie, A.M., Frith, U. 1985. Does the autistic child have a "theory of mind? Cognition, 21, 37-46.

Baron-Cohen, S. 1995. Mindblindness. Cambridge: MA: MIT Press.

Baron-Cohen, S. 2000. Theory of mind in autism: a fifteen-year overview. In: Baron-Cohen, S., Tager-Flusberg, H., Cohen, D.J. (Eds.). Understanding Other Minds perspectives from developmental cognitive neuroscience. Oxford: Oxford University Press.

Chomsky, N. 1968. Language and Mind. New York. Harcourt.

Frith, Ch.D., Wolpert, D.M. 2003. The Neuroscience of Social Interaction: Decoding, imitating, and influencing the actions of others. Oxford University Press.

Frith, Ch. 2007. Making up the Mind: How the Brain Creates Our Mental World. Blackwell Publishing.

Happé, F. 2003. Theory of mind and the self. In Annals Of The New York Academy Of Sciences, 1001 (1): 134-144.

Paradis M. 2009. Cerebral divison of labour in verbal communication. In: Cognition and Pragmatics. Dominek S. - J. Östman, and J. Vershueren (Eds.) John Benjamins.

Perkins M. 2010. Pragmatic Impairment. Cambridge University Press. 